\title{
De las leyes isométricas a las leyes alométricas: enseñanza interdisciplinar de las Ciencias Naturales utilizando una Metodología de Aprendizaje Activo modificada a la virtualidad
}

\section{From isometric to allometric laws: interdisciplinary teaching of Natural Sciences using an Active Learning Methodology modified to virtuality}

\author{
VILLA CELEDON, Ovidio ${ }^{1}$ \\ MONROY-RAMIREZ, Freddy A. ${ }^{2}$
}

\begin{abstract}
Resumen
Este artículo presenta los resultados de una estrategia de enseñanza en Ciencias Naturales con estudiantes de secundaria de la Institución Educativa CASD Simón Bolívar en Valledupar, Colombia. La temática inició desarrollando conceptos fundamentales como factores de escala, leyes isométricas y alométricas, hasta llegar a la ley de Kleiber y algunas aplicaciones. Su enfoque experimental, conjugó interdisciplinariamente áreas como Matemáticas, Biología y Física. Se utilizó la Metodología de Aprendizaje Activo modificada a la virtualidad como marco didáctico durante la pandemia.

Palabras clave: leyes isométricas, leyes alométricas, ley de kleiber, enseñanza virtual
\end{abstract}

\begin{abstract}
This paper presents the results of a teaching strategy in Natural Sciences with high school students from the Educational Institution CASD Simón Bolívar in Valledupar, Colombia. The topic started by developing fundamental concepts such as scale factors, isometric and allometric laws, until reaching Kleiber's law and some applications. Its experimental approach interdisciplinary combined areas such as Mathematics, Biology and Physics. The Active Learning Methodology modified to virtuality was used as a didactic framework during the pandemic.

Key words: isometric laws, allometric laws, kleiber's law, virtual teaching
\end{abstract}

\section{Introducción}

De la geometría elemental se sabe que dos figuras geométricas son semejantes aun cuando sus tamaños puedan ser diferentes, sus formas se conservan iguales y se puede establecer alguna proporción aritmética entre algunos

\footnotetext{
${ }^{1}$ Professor Department of Basic Sciences. Universidad Popular del Cesar. Valledupar, Colombia. Email: ovidiovilla@unicesar.edu.co.

${ }^{2}$ Retired Tenured Professor. Physics Department. National University of Colombia, Bogotá, Colombia. Email: famonroyr@unal.edu.co.
} 
de sus elementos (Pogorelov, 1974) por ejemplo, para un cuadrado: entre sus lados o entre sus áreas, para un cubo entre sus lados, entre las áreas de sus caras o entre sus volúmenes, para una esfera entre sus radios o sus volúmenes, esto debido al Principio de Semejanza Geométrica (Guzman Peña, 2019), el cual permite el planteamiento de la Ley Isométrica (Gutierrez Sanchez \& Sanchez Garduño, 2017); sin embargo, al cuestionarse, si esta ley isométrica es válida para cualquier figura geométrica, como por ejemplo un cubo o un cono, no se cumplen, ya que para conservar la semejanza de las figuras es necesario el cambio de dos de sus variables simultáneamente, el diámetro y la altura. Este hecho evidencia la necesidad del planteamiento de las Leyes Isométricas (Perez \& Fernandez, 2015) con el uso del Modelo de Semejanza Elástica (Guzman Peña, 2019), (Villa Celedon, 2020) que relaciona dos variables geométricas (por ejemplo altura y diámetro) mediante exponentes no enteros; no obstante, estas leyes también tienen un origen meramente geométrico. Cuando se desea aplicar estas Leyes Isométricas a otros campos de las ciencias, por ejemplo la Biología, es necesario el planteamiento de una hipótesis biológica, que permita establecer relaciones concretas entre las variables geométricas y alguna función o característica de los seres vivos. Estas consideraciones posibilitan el planteamiento de las llamadas Relaciones Alométricas, las cuales relacionan típicamente las variables geométricas con la masa del órgano o del cuerpo completo del ser vivo. Cuando en Biología se quiere describir la morfometría de los seres vivos (el crecimiento de las diferentes partes del cuerpo mismo), se observa que no todas las partes del cuerpo crecen al mismo ritmo o no en todos los animales de una misma especie (macho o hembra) sus órganos crecen al mismo ritmo (Mirth, Frankino, \& Shingleton, 2016) (Zaballos, Diaz, \& Garcia Moreno, 2009), a esta parte de la Biología se le llama Alometría (Gutierrez Sanchez \& Sanchez Garduño, 2017), (Sanchez Garduño \& Gutierrez Sanchez, 2020), (Gould, 1966), (Zaballos, Diaz, \& Garcia Moreno, 2009).

Para la aplicación de las leyes alométricas a problemas concretos en Biología, los estudiantes de secundaria deben acudir al trabajo con exponentes enteros y fraccionarios, plantear hipótesis biológicas e hipótesis físicas, lo cual es uno de los objetivos fundamentales del presente trabajo: evidenciar la necesidad de la interdisciplinariedad. No obstante, para motivar a este estudio interdisciplinar de las Ciencias es necesaria la implementación de alguna estrategia motivadora, que para el caso será mayormente experimental, con la utilización de elementos de bajo costo y fácil consecución (para este caso, papel, cartulina, plastilina). La estrategia elegida fue la Metodología de Aprendizaje Activo (MAA) (Sokoloff \& Thornton, 1997), (Lakhdar, Culaba, Lakshminarayanan, \& Maquiling, 2006), la cual cumple con los requerimientos del trabajo inicialmente planeado presencialmente; para tal fin se plantearon varias prácticas de cuantificación de áreas y volumenes con el uso de cartón y plastilina y sus respectivos cálculos numéricos, para estimar posteriormente los tamaños de animales y partes de sus cuerpos o personas de distintas tallas que guardan semejanzas. Después de esto se realizaron algunas aplicaciones relacionadas directamente con la Biología, tales como la fuerza relativa de los animales y la división celular con el uso de las relaciones alométricas. Posterior a esta práctica y usando el Modelo de Semejanza Elástica y la aproximación a forma cilíndrica de algunos huesos de animales y troncos de árboles, se describieron sus crecimientos. Finalmente, se realizaron algunas aplicaciones del planteamiento de una hipótesis biológica, las Relaciones Alométricas y la Tasa Metabólica Basal, para llegar a la Ley de Kleiber.

El resultado del trabajo de los estudiantes se midió por medio de la comparación entre las pruebas de entrada y salida utilizando el Factor de Ganancia Normalizada de Hake (GNH) (Hake, 1998), con 18 estudiantes del grado octavo de la Institución Educativa CASD Simón Bolívar de la ciudad de Valledupar, departamento del Cesar (Colombia).

Dado que este trabajo se realizó entre los años 2019 y 2020 hubo necesidad de modificar los pasos de la MAA para que el componente experimental se realizará completamente, pero de manera virtual, debido a la pandemia que apareció mundialmente por el Coronavirus SARS-CoV-2 que obligó al aislamiento obligatorio preventivo en muchos países del mundo. 


\section{De las leyes isométricas a las leyes Alométricas}

De la geometría elemental se conoce que la razón entre los lados homólogos de dos figuras geométricas semejantes es una constante numérica denominada factor de escala $k$. Si la proporción se realiza entre sus áreas, dicha constante se convierte en $k^{2}$ y si se realiza entre sus volúmenes es $k^{3}$, así:

Figura 1

(a) Magnificación de un cuadrado de lado L por L'= kL,

(b) Magnificación de un cubo de lado L por L'=kL, (c) Magnificación de una circunferencia de radio $\mathrm{r}$ por $\mathrm{r}^{\prime}=\mathrm{kr}$,

(d) Magnificación de una esfera de radio $\mathrm{r}$ por $\mathrm{r}^{\prime}=\mathrm{kr}$.

(a)

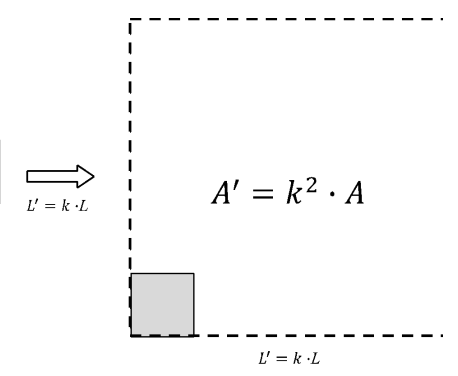

(c)

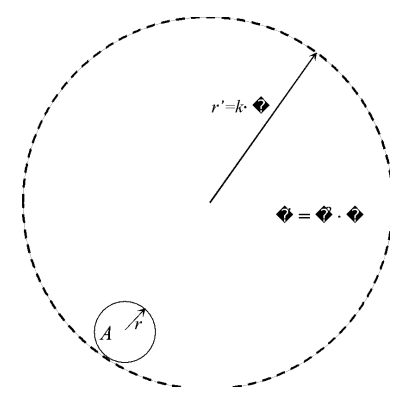

(b)

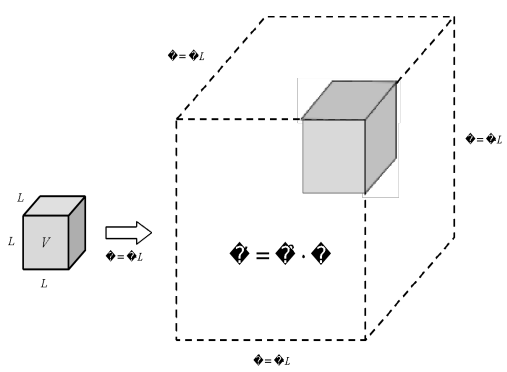

(d)

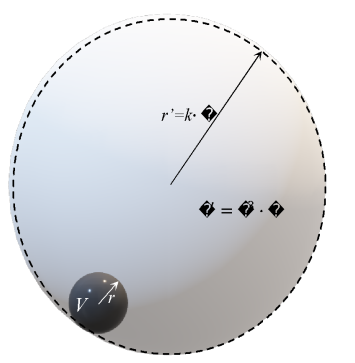

Fuente: (Villa Celedon, 2020)

A las relaciones geométricas $\frac{L^{\prime}}{L}=k, \frac{A \prime}{A}=k^{2}$ y $\frac{V^{\prime}}{V}=k^{3}$ se les denomina Relaciones Isométricas, en estas, basta con cambiar la longitud de una de las magnitudes de una figura geométrica y se obtiene otra figura semejante (Gutierrez Sanchez \& Sanchez Garduño, 2017), sin embargo, esto no sucede con todas las figuras geométricas, como por ejemplo el cilindro y el cono. 


\section{Figura 2}

(a) Cilindros con diferentes radios y diferentes alturas, (b) Conos con diferentes radios y diferentes alturas; no son figuras semejantes porque su forma cambia, al cambiar simultáneamente solo una de sus magnitudes; (c) Cilindros semejantes: su altura y radio varían simultáneamente; (d) Conos semejantes: su altura y radio varían simultáneamente.

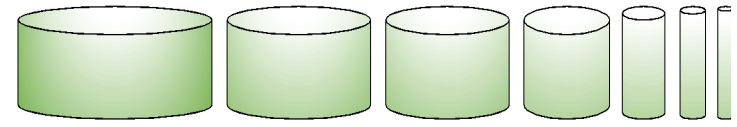

(a)

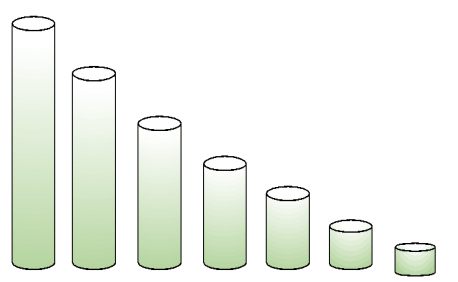

(c)
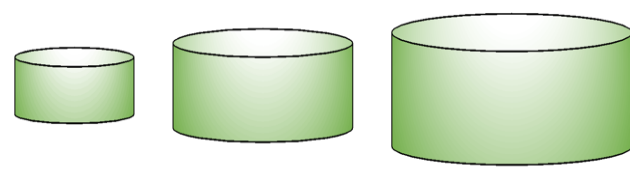

(d)
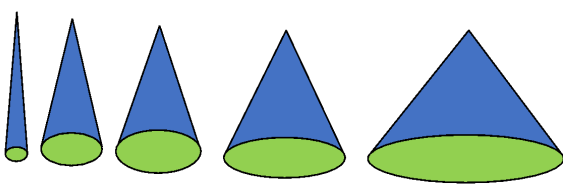

(b)
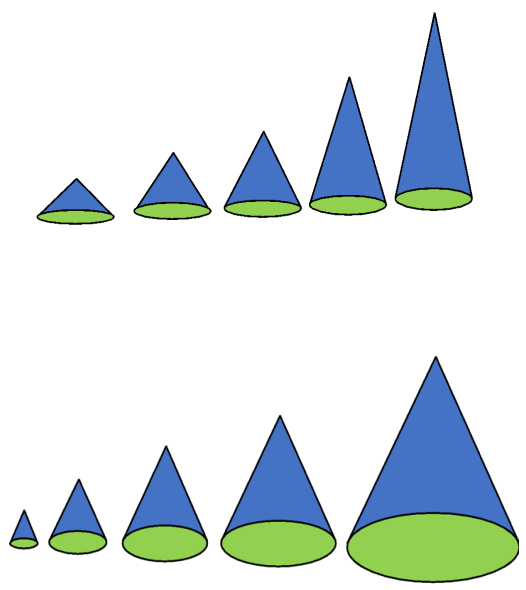

Fuente: (Villa Celedon, 2020)

En los cilindros y conos para que se mantenga la forma (aunque cambie su tamaño), es necesario cambiar simultáneamente dos variables, por ejemplo la altura $(L)$ y el diámetro $(d)$, lo cual según el Modelo de Semejanza Elástica (MSE) se consigue mediante la relación empírica $d=L^{\frac{3}{2}}$ (Guzman Peña, 2019). En el caso de un cilindro, cuando se relaciona el MSE con la masa (asumiendo distribución de masa uniforme), se pueden obtener las llamadas Relaciones Alométricas (RA), las cuales pueden ser usadas para comparar dos estructuras cilíndricas de longitudes $L$ y $L^{\prime}$, diámetros $d$ y $d^{\prime}$, superficies $S$ y $S^{\prime}$ y masas $M y M^{\prime}$, como por ejemplo dos árboles o dos huesos cuya forma se pueda aproximar a un cilindro, como se muestra en la figura 3 :

\section{Figura 3}

Estructuras semejantes cuya forma se puede aproximar a cilindros y aplicar el modelo de semejanza elástica.
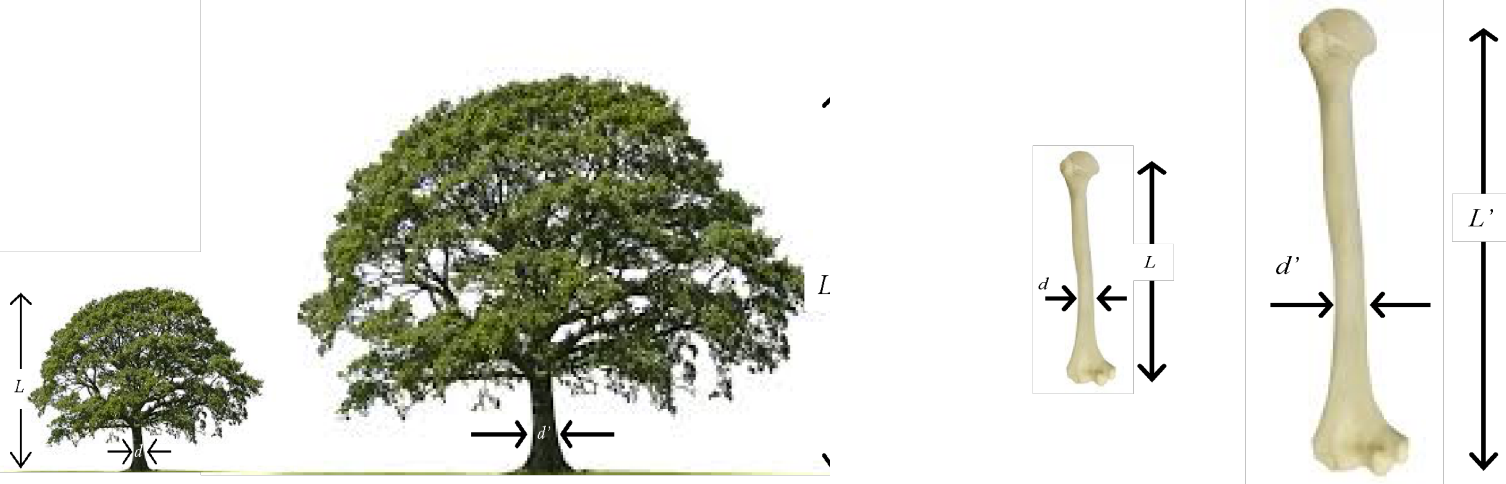

Fuente: (Villa Celedon, 2020) 
Algunas de las $R A$ se pueden expresar como (Gould, 1966):

Relación entre longitudes y masas

Relación entre diámetros y masas

Relación entre superficies y masas

$$
\frac{L^{\prime}}{L}=\left(\frac{M^{\prime}}{M}\right)^{\frac{1}{4}}
$$$$
\frac{d^{\prime}}{d}=\left(\frac{M^{\prime}}{M}\right)^{\frac{3}{8}}
$$

$$
\frac{S^{\prime}}{S}=\left(\frac{M^{\prime}}{M}\right)^{\frac{5}{8}}
$$

Estas relaciones, junto con el planteamiento de una hipótesis biológica pertinente, según el caso, conforman la Alometría.

La Alometría es la parte de la Biología que estudia los cambios diferenciados en el crecimiento de las partes del cuerpo entre sí y con el mismo cuerpo como un todo (Sanchez Garduño \& Gutierrez Sanchez, 2020), (Villa Celedon, 2020). Del mismo modo analiza aquellos procesos biológicos que se modifican en un cuerpo con el cambio de sus dimensiones (Gould, 1966), es decir, su relación con las variables que permiten describir cuantitativamente algunos procesos fisiológicos del ser, debido a que los cambios de forma responden a factores de tipo funcional (Sanchez Garduño \& Gutierrez Sanchez, 2020).

Las leyes que rigen la Alometría parten de una relación potencial de la forma:

$$
y=a_{0} x^{b}
$$

Donde $a_{0}$ y $b$ son constantes, $x$ e $y$ son las variables asociadas a las dimensiones y/o funciones biológicas, como por ejemplo $y$ la magnitud del órgano de crecimiento diferencial, $x$ el tamaño del cuerpo que se toma como estructura de referencia (normalmente una medida global del tamaño del organismo), $b$ es la razón de crecimiento diferencial de una parte del cuerpo con respecto a la rata de crecimiento del cuerpo (Zaballos, Diaz, \& Garcia Moreno, 2009). La ecuación (2) se puede reescribir linealmente como:

$$
Y=A_{0}+b \cdot X
$$

Con $Y=\log (y), X=\log (x)$ y $A_{0}=\log \left(a_{0}\right)$. El valor del exponente $b$ (no necesariamente número entero) de la ecuación (2), (pendiente de la recta en la ecuación (3)) determina si hay isometría o alometría: si $b=1$ la forma se mantiene al cambiar el tamaño, por lo tanto es isometría, si $b>1$ es llamada alometría positiva porque $Y$ crece más rápidamente que $X$ y si $b<1$. Se llama alometría negativa, porque $Y$ crece más lento que $X$ (Zaballos, Diaz, \& Garcia Moreno, 2009).

\section{Ley de Kleiber}

Una de las definiciones del Metabolismo Basal $(M B)$ se refiere a la cantidad de energía mínima que necesita un organismo vivo en reposo para sobrevivir realizando las funciones básicas, como respirar, bombear el corazón, filtrar la sangre, sintetizar hormonas o parpadear (Nuñez, Calderon, \& Gil, 2010). Este MB se mide por medio de 
la Tasa Metabólica Basal (TMB) en unidades de kilocalorías por día: kcal/día, como la cantidad de energía por día que necesita el ser vivo para sobrevivir (Nuñez, Calderon, \& Gil, 2010), (Hulbert \& Else, 2004). Históricamente se han planteado varias hipótesis biológicas para relacionar las dimensiones de los cuerpos con la TMB (Hudson, Isaac, \& Reuman, 2013), (Sanchez Garduño \& Gutierrez Sanchez, 2020), sin embargo, una de las que más se acerca a la realidad es aquella que supone que la $T M B$ es proporcional al área de la sección transversal promedio del hueso o del grosor promedio del cuerpo (o parte de él) del ser vivo: $T M B: \propto: S_{T}$. Esta hipótesis biológica permitió afinar el exponente de la masa al relacionarla con la $T M B$ ya que se acercaba mucho más a la realidad de muchos mamíferos.

\section{Figura 4}

Para algunas especies, la sección transversal de sus cuerpos o partes de ellos, o de sus extremidades, puede aproximarse a circunferencias. (Villa Celedon, 2020).
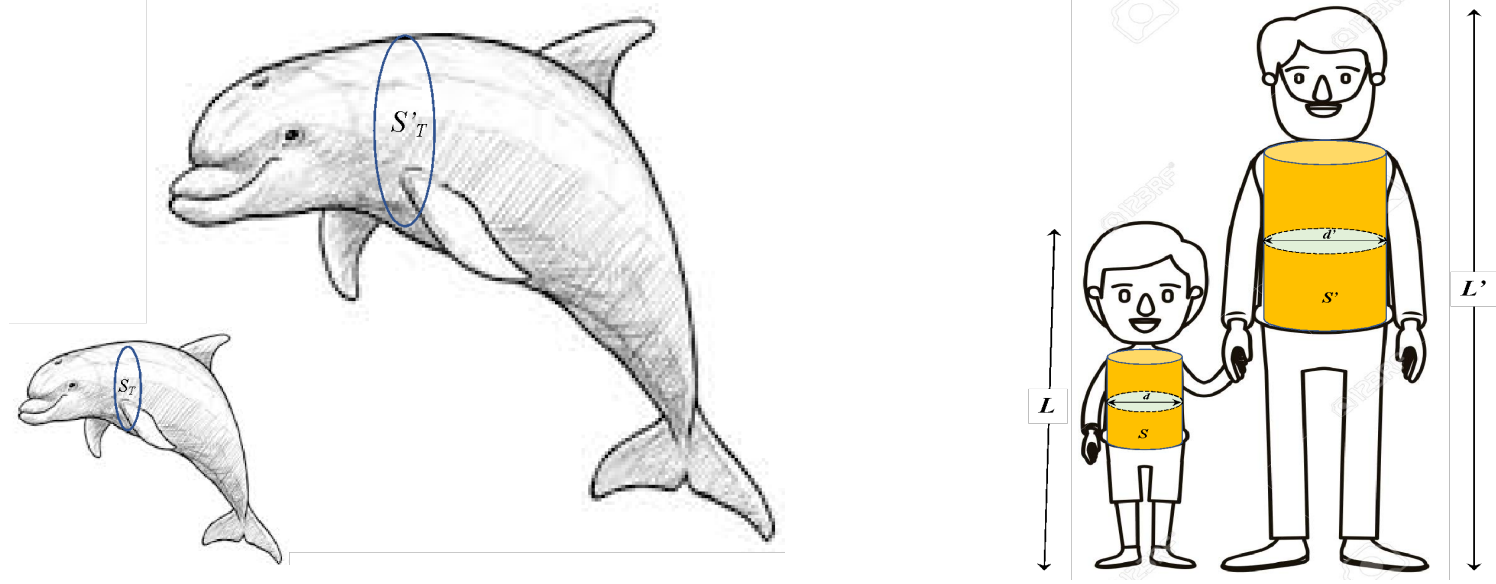

Fuente: (Villa Celedon, 2020)

Si la sección transversal promedio $\left(S_{T}\right)$ es poco más o menos una circunferencia de diámetro $d: S_{T}=\frac{\pi}{4} d^{2}$, asumiendo que se puede aproximar, en promedio, una parte del cuerpo a un cilindro, se cumple la $R A d: \propto: M^{\frac{3}{8}}$, por lo tanto, se tiene que: $T M B: \propto: M^{\frac{3}{4}}$, es decir que para dos cuerpos de masas $M y M^{\prime}$, de tasas Metabólicas Basales $T M B$ y $T M B^{\prime}$ 'respectivamente, se tiene que:

$$
\frac{T M B^{\prime}}{T M B}=\left(\frac{M^{\prime}}{M}\right)^{\frac{3}{4}}
$$

La ecuación (4) se denomina Ley de Kleiber y es la ecuación cuyo exponente es el más cercano a la realidad de muchas especies (Sanchez Garduño \& Gutierrez Sanchez, 2020).

\section{Estrategia didáctica basada en la Metodología de Aprendizaje Activo modificada a la virtualidad (MAA-MV)}

La MAA tradicional exige la presencialidad de los estudiantes y docentes para la realización de los experimentos en los que está basada, ya que en ella el experimento es el protagonista dentro del proceso de enseñanzaaprendizaje y permite la construcción del conocimiento a través de la observación directa del mundo real. La estructura típica de la MAA se puede resumir en: (Sokoloff \& Thornton, 1997), (Lakhdar, Culaba, Lakshminarayanan, \& Maquiling, 2006): 
1. El docente describe y plantea una situación (no necesariamente un problema).

2. Cada estudiante registra por escrito sus predicciones.

3. Se discuten las predicciones individuales en grupos.

4. Se registra el resumen de las predicciones grupales.

5. Se socializa para todos el resumen de las predicciones.

6. Se realiza el experimento mostrando claramente los resultados. Si lo realiza el profesor es práctica demostrativa $(P D)$, si lo realizan los estudiantes es práctica interactiva $(P I)$.

7. Se discuten y registran los resultados de cada grupo.

8. Se realiza la síntesis de los conceptos involucrados en la práctica, se extrapolan los resultados y los estudiantes escriben sus conclusiones.

Dada la enseñanza virtual a la que nos vimos abocados en el año 2020 y con el fin de mantener la comunicación con los estudiantes, fue necesario realizar algunas modificaciones a la MAA tradicional usando varias plataformas de comunicación sincrónica y asincrónica tales como Zoom ${ }^{\circledR}$, Google meet ${ }^{\circledast}$, WhatsApp ${ }^{\circledR}$ y correo electrónico. Con el ánimo de mantener la misma estructura mencionada anteriormente, pero ahora en enseñanza virtual, todos los pasos se desarrollaron virtualmente con el uso de estas plataformas, pero la mayor modificación se hizo en el paso 6, así:

6a. Sincrónicamente y con tiempo limitado, estando conectados todos los estudiantes y el profesor al mismo tiempo, cada estudiante resolvió las actividades descritas en la guía, evidenciando y consultando las partes en las que tenía dudas o ignoraba.

6b. Sincrónicamente y con tiempo limitado por comunicación virtual se compartieron los resultados y las dudas en grupos de máximo 3 estudiantes.

Luego se prosiguió con los demás puntos desde el 7 en adelante.

La Metodología de Aprendizaje Activo (MAA) es una de las metodologías enmarcadas dentro del constructivismo y válida cuando se desea que el experimento sea el protagonista en el proceso de enseñanza-aprendizaje, ya que permite la construcción del conocimiento a través de la observación directa del mundo real. Su estructura se puede resumir en los siguientes pasos, denominados PAODS (Sokoloff \& Thornton, 1997), (Villa Celedon, 2020):

Predicción: sobre lo que puede suceder ante el planteamiento de una situación determinada.

Actividad: realización de la actividad propuesta.

Observación: atenta y activa de los resultados.

Discusión: de los resultados y confrontación con las predicciones.

Síntesis: y formalización de los conceptos o relaciones estudiadas entre variables (en el mejor de los casos realizada por los estudiantes).

En la MAA cada estudiante es el eje central en el desarrollo de sus capacidades para desarrollar la crítica, fomentar el pensamiento independiente, el trabajo colaborativo, el descubrimiento experimental en forma directa, el trabajo individual y grupal y el debate (Sokoloff \& Thornton, 1997), (Villa Celedon, 2020).

A la MAA con estas adiciones permanentes en todo su proceso, se le dio el nombre de MAA modificada a la virtualidad $M A A-M V$. 


\section{Principales actividades desarrolladas}

\subsection{Procedimiento}

La estrategia se diseñó en el orden siguiente: primero se realizó una prueba de entrada, posteriormente se desarrollaron las cinco guías que contenían cada una de ellas, varias actividades teórico-prácticas y al final de cada una de ellas una evaluación formativa. Para cerrar el proceso se implementó una evaluación final. Para el análisis cualitativo de los resultados se tuvo en cuenta el resultado de todas las evaluaciones: diagnóstica, formativa y sumativa; para análisis cuantitativo se compararon los resultados de las evaluaciones inicial y final por medio de la ganancia normalizada de Hake (Villa Celedon, 2020).

\subsection{Descripción}

Con la finalidad de evaluar la suficiencia de los preconceptos con los que llegan los estudiantes al curso, e identificar los posibles temas que requieren refuerzo, se diseñó y aplicó una prueba de entrada (o diagnóstica) de diez preguntas en forma de test de selección múltiple con única respuesta. En esta prueba de entrada las primeras cuatro preguntas exploraron el tema de áreas y volúmenes de figuras geométricas simples y combinadas, así como la distribución de volúmenes grandes en volúmenes pequeños. La pregunta cinco exploró la variación de una variable con el cambio de otra en un volumen. La pregunta seis exploró la capacidad de los estudiantes de estimar los valores o rangos de algunas magnitudes fundamentales. Las preguntas siete a diez exploraron los posibles preconceptos sobre fuerza relativa de algunos animales, las cuales se podrían resolver por el cálculo de una regla de tres simple directa.

Para el desarrollo del tema central de la estrategia, se diseñaron y aplicaron cinco guías interactivas enmarcadas dentro de la MAA. En todas las guías, además de respetar todos los pasos de la $M A A-M V$, al final se realizó una evaluación por medio del planteamiento de una situación similar a la desarrollada en la respectiva guía. La primera guía se realizó sobre estimación de magnitudes, con la finalidad de ayudar a los estudiantes a ubicarse dimensionalmente en la estimación de diversas magnitudes a distintas escalas y evidenciar sus diferencias y semejanzas; también se propusieron actividades que orientaban el cálculo de volúmenes y distribución de un volumen dentro de otro. En la segunda guía se desarrolló el cálculo de volúmenes de cuerpos sólidos, así como también la estimación del volumen de algún animal, partiendo del cálculo de volúmenes de figuras geométricas regulares que los podían aproximar, como cilindros, esferas, etc. En la tercera guía se desarrollaron actividades encaminadas a evidenciar la existencia de los factores de escala netamente geométricos (con cubos, esferas y cilindros: modificando simultáneamente su altura y diámetro): relación de longitudes $k$, de áreas $k^{2}$ y de volúmenes $k^{3}$.

La figura 5 muestra el pantallazo de algunos estudiantes resolviendo las evaluaciones de las guías 3 y 4.

En la cuarta guía se orientaron actividades que llevaron al estudiante a la aplicación de las relaciones isométricas, utilizando la morfología de algún animal y algunas relaciones físicas y biológicas, tales como la fuerza relativa de los animales y la división celular; esto reforzó notablemente el concepto de interdisciplinariedad (entre matemáticas, biología y física) que se le quería dar al trabajo. Partiendo del conocimiento de las relaciones isométricas, y utilizando la simetría cilíndrica, en la quinta guía se planteó el Modelo de Semejanza Elástica y se ambientó con la aplicación a problemas concretos del crecimiento de árboles y de algunos huesos de animales, aproximándose a cilindros. Para deducir las Relaciones Alométricas, se plantearon algunas hipótesis biológicas como por ejemplo la relación del diámetro con la masa, la relación de la superficie corporal con la masa y, con la definición de la $T M B$, se planteó la Ley de Kleiber. Todo lo anterior aplicado a características geométricas y funciones biológicas de algunos animales. 
Figura 5

Captura de pantalla del trabajo virtual con los estudiantes durante la retroalimentación de la evaluación de las guías 3 y 4.
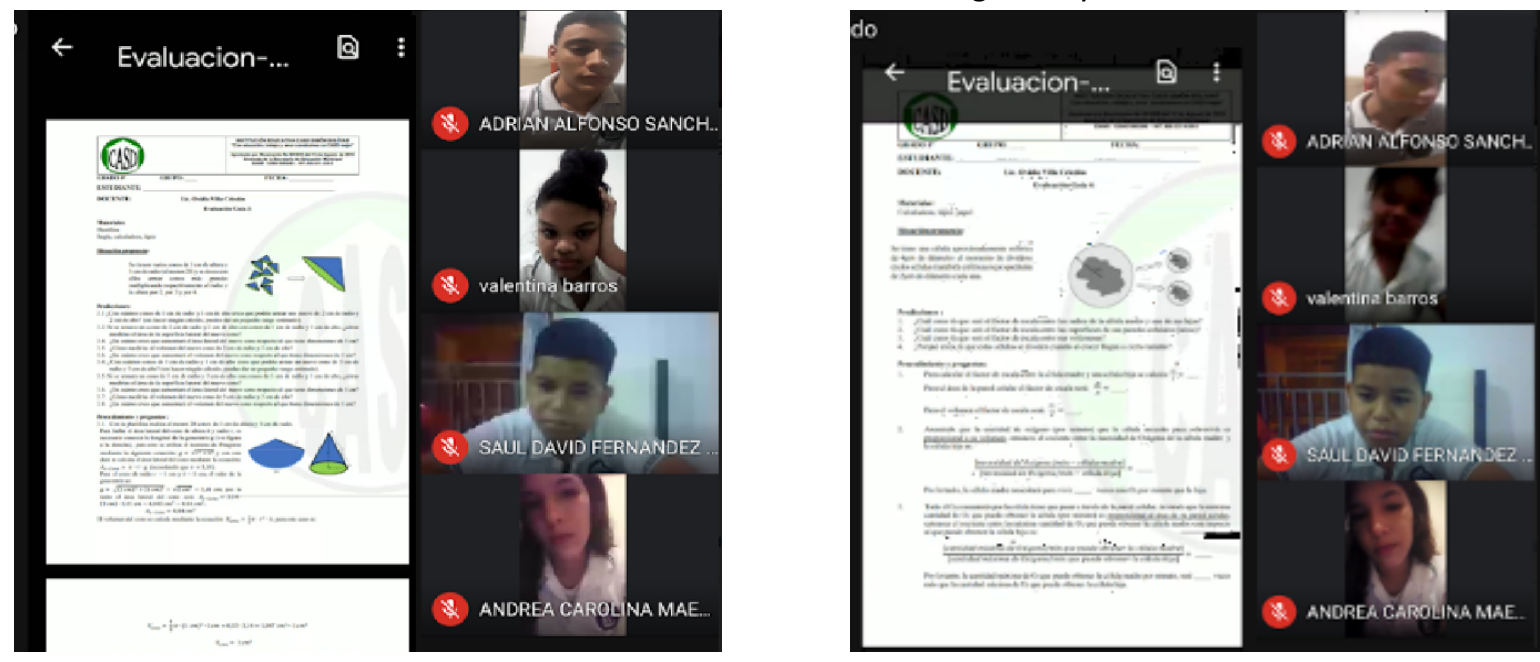

Fuente: (Villa Celedon, 2020)

La evaluación final se implementó con diez preguntas en forma de selección múltiple con única respuesta. Las primeras cinco preguntas exploraron temas de varias de las preguntas de la evaluación inicial, con preguntas similares, pero no idénticas con el propósito de comparar los resultados entre las dos evaluaciones. Las cinco preguntas restantes exploraron los mismos temas de las evaluaciones de las cinco guías con la finalidad de verificar el avance al final con respecto a todas las guías desarrolladas (Villa Celedon, 2020).

La figura 6 muestra un pantallazo de algunos estudiantes resolviendo la evaluación final, la cual también fue realizada virtualmente, pero de manera sincrónica y con tiempo limitado.

Figura 6

Captura de pantalla de los estudiantes resolviendo la evaluación final.

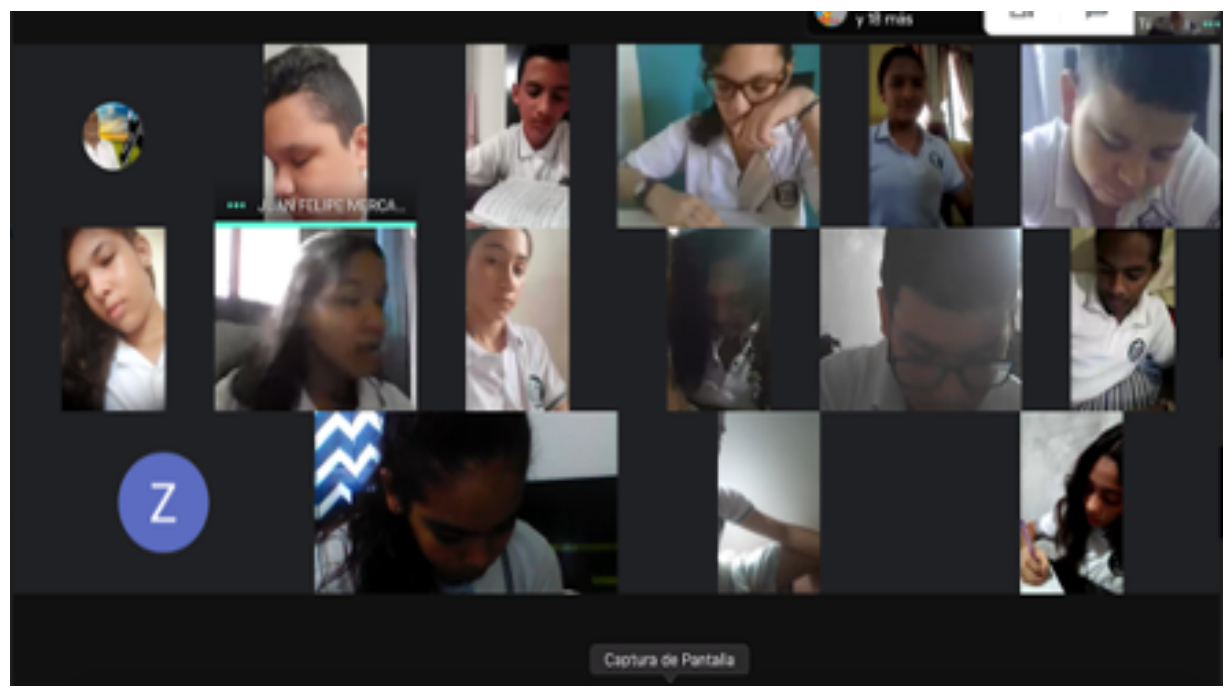

Fuente: (Villa Celedon, 2020) 


\section{Resultados y discusión}

Según se describió en la sección anterior, las primeras cuatro preguntas de la evaluación inicial exploraron preconceptos sobre cálculo de áreas y volúmenes de figuras regulares, según se observa en la gráfica de la figura 7. Los resultados obtenidos en estas preguntas no fueron muy positivos, pero en la tercera y cuarta pregunta mejoraron notoriamente; sin embargo, en la pregunta cinco, donde se exploró sobre relación entre variables, el resultado fue bastante deficiente, ya que menos del $6 \%$, (lo cual equivale a solo un estudiante de los 18 que respondieron), respondió bien a esta pregunta. Según los resultados mostrados en la gráfica de la figura 7, los resultados en las respuestas a las demás preguntas fueron excelentes, excepto en la pregunta 9, la cual indagaba sobre la relación de una variable con el cuadrado de la otra. Esta pregunta no fue respondida correctamente por ningún estudiante, lo cual podría ser evidencia de que la pregunta pudo ser muy compleja para ellos o tuvieron alguna confusión en la aritmética, dado que en este caso particular, no es una regla de tres simple directa linealmente, sino con el cuadrado de una de las variables. El porcentaje general de aciertos en esta prueba fue del 52\%, lo cual claramente muestra una deficiencia promedio del grupo en los temas evaluados.

La figura 7 muestra los resultados generales obtenidos por los 18 estudiantes en la prueba de entrada.

Figura 7

Resultados de la evaluación de entrada.

Resultados Evaluación Diagnóstica

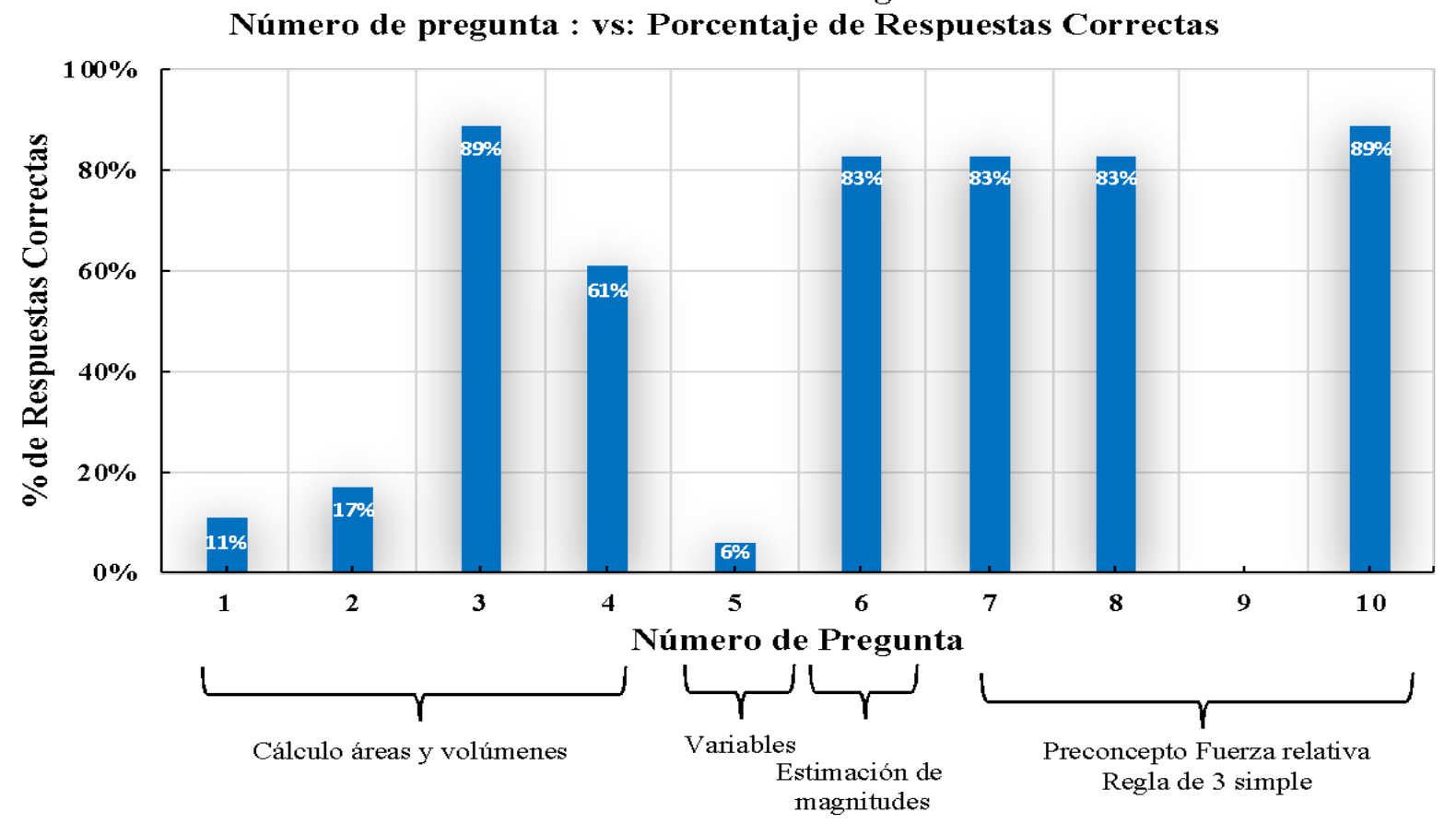

Fuente: (Villa Celedon, 2020)

En la evaluación de la guía 1, en las preguntas 1 y 2 se exploró el estimativo y cálculo de volúmenes combinando geometrías cúbicas y esféricas y la estimación y cálculo del número de objetos (cúbicos y esféricos) a distribuir en un volumen. En la pregunta 3 se pidió hacer una justificación sobre las diferencias entre la estimación y el cálculo realizado, considerando el cálculo de los volúmenes y su distribución en un volumen mayor. Esta última pregunta requiere hacer argumentaciones sobre el volumen que ocupan los cuerpos geométricos en el espacio y la forma de minimizar su distribución espacial en un volumen fijo (como introducir el máximo número de dulces esféricos y cúbicos en una taza cilíndrica). La figura 8 muestra los resultados que se cuantificaron a las respuestas 
a estas tres preguntas (aunque inmersas en estas tres preguntas, habían varias preguntas abiertas, cuyas respuestas no se cuantificaron).

Figura 8

Resultados evaluación guía 1

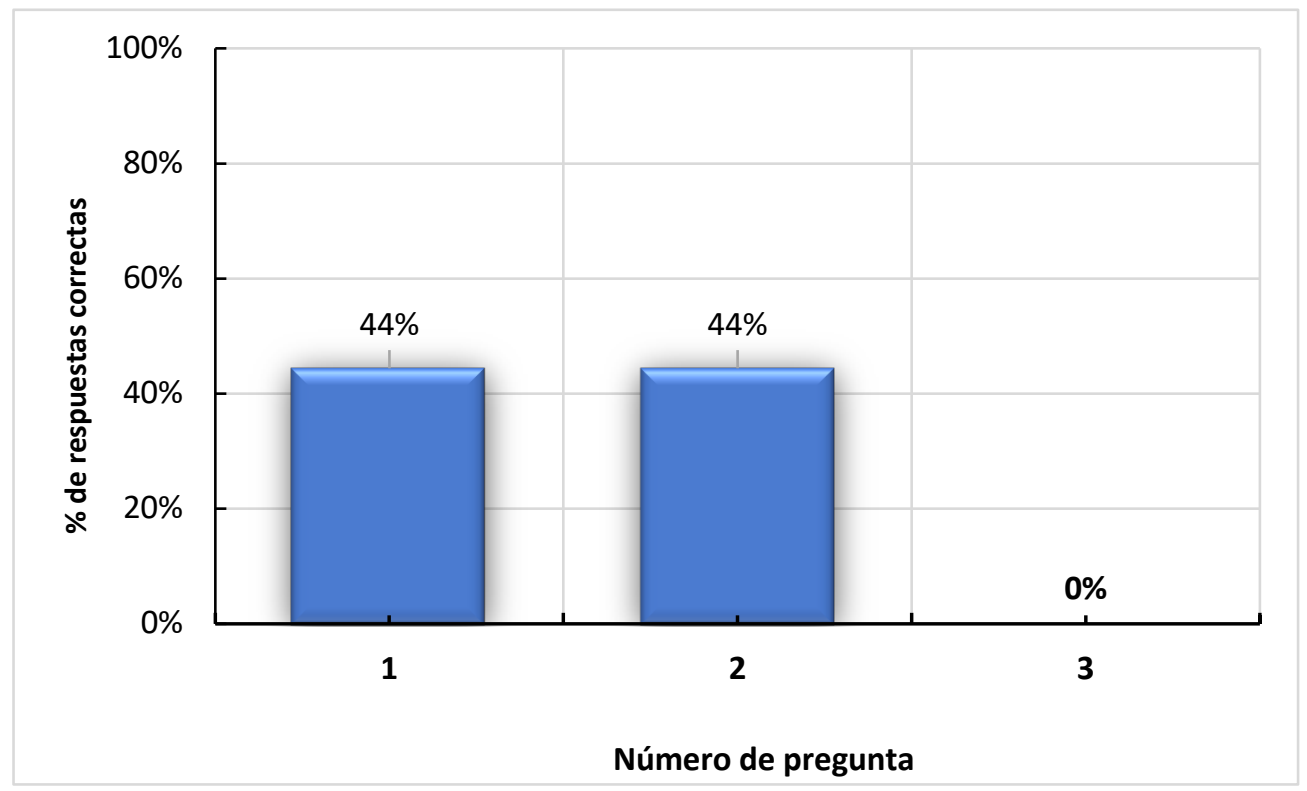

Fuente: (Villa Celedon, 2020)

Observando estos resultados, 8 de los 18 estudiantes respondieron correctamente a las preguntas de estimativo y cálculo; pero la tercera pregunta donde se requiere alguna argumentación, ningún estudiante respondió correctamente. Este hecho permite afirmar que no solamente no están acostumbrados a hacer este tipo de cálculos rápidos de volúmenes mezclados, sino que carecen de experticia en la construcción de argumentos. Estas dificultades bien podrían relacionarse con el nivel académico y edades, donde son muy comunes este tipo de yerros. No obstante, es importante señalar que se debe fortalecer este trabajo, para que poco a poco los estudiantes vayan aprendiendo a estimar valores, o al menos rangos de algunas magnitudes fundamentales 0 sus derivadas, así como avanzar en la construcción de argumentos (Villa Celedon, 2020).

En la evaluación de la guía dos, además de varias preguntas abiertas y de respuestas cualitativas sobre estimación de volúmenes de animales, se hicieron cinco preguntas que requerían de una respuesta cuantitativa de cálculo de suma de volúmenes de figuras regulares (cilindros, esferas), a las cuales se podían hacer varias aproximaciones al volumen de un animal. En particular, la pregunta cinco (que obtuvo $0 \%$ de respuestas correctas) probablemente requiere de más análisis y manejo de los resultados obtenidos en los cálculos numéricos. Por ejemplo, a partir del cálculo del volumen de los animales, identificar cuántas veces cabría aproximadamente uno en el otro (aunque podrían haberlo hecho dividiendo el volumen del animal grande entre el volumen del animal pequeño).

La figura 9 muestra los resultados cuantificados para la evaluación de la guía 2. 
Figura 9

Resultados evaluación guía 2.

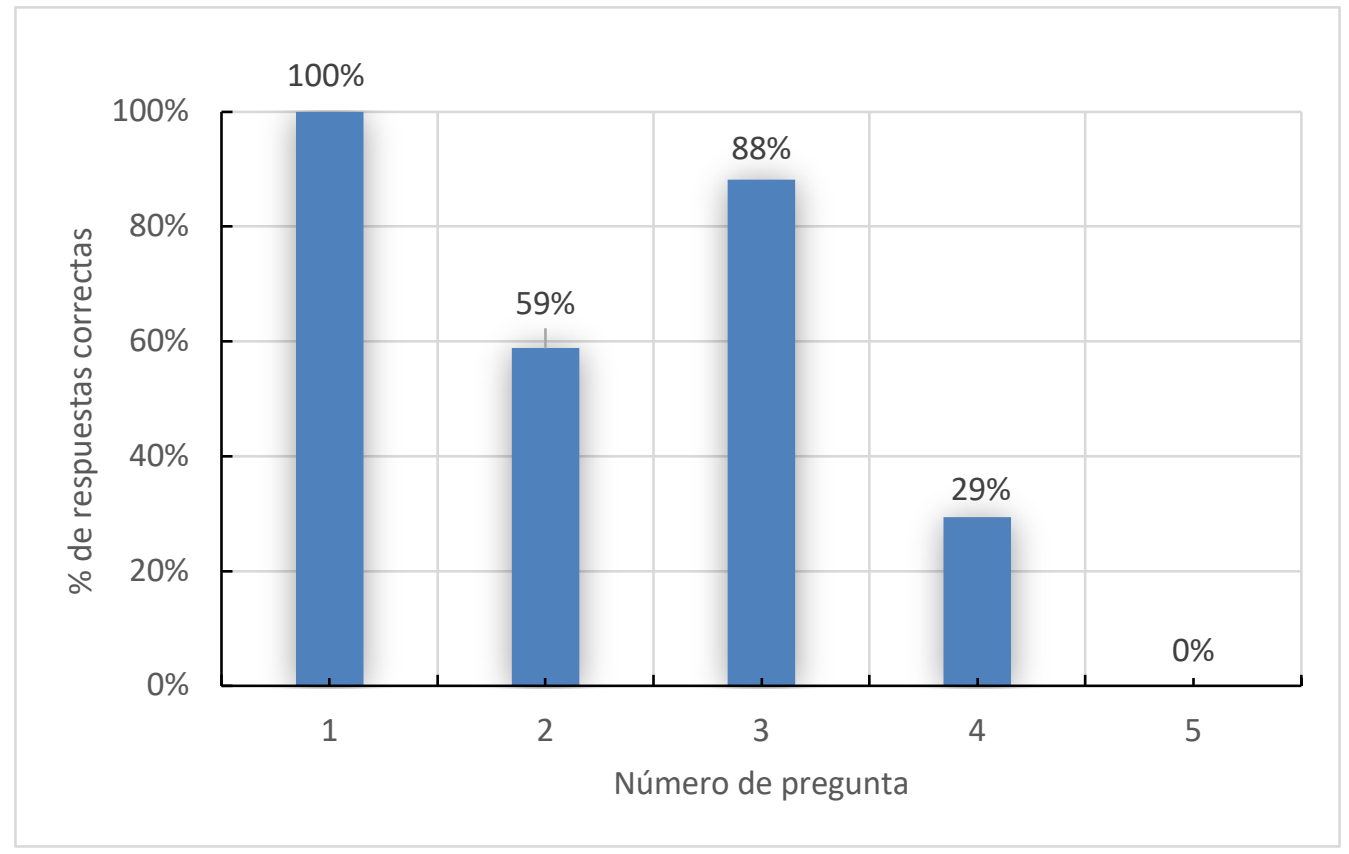

Fuente: (Villa Celedon, 2020)

De la figura anterior es claro que la mayoría de estudiantes no tuvieron mucha dificultad en responder a las primeras cuatro preguntas; sin embargo, en la pregunta cinco que requiere del análisis y argumentación de la respuesta, todos fallaron. Esto puede ser debido a que los estudiantes no tienen mucha idea de las dimensiones relativas de los animales de muy diferentes tamaños, pese a esto, este proceso es necesario para aprender a hacer mejores estimativos (al menos en volumen, como en este caso). Aunque el promedio aritmético de las calificaciones obtenidas por los estudiantes en esta guía fue del $28 \%$ de acierto, este resultado no es muy diciente ya que, además de haberse encontrado los dos extremos de porcentaje de acierto (100\% y $0 \%)$, el análisis cualitativo de los estudiantes en las preguntas aporta mayor infomación sobre su avance (Villa Celedon, 2020).

En cuanto a la evaluación de la guía tres se refiere, se trabajó con un cono, ya que este requiere la variación simultánea de dos variables (radio y altura) para conservar su semejanza. En esta evaluación de la guía 3 se mezclaron los pasos del procedimiento a seguir con algunas preguntas; por esta razón, se presenta una complementariedad entre la parte numérica (que permite la evaluación cuantitativa) y la parte teórica (que permite una evaluación cualitativa, ya que, además de realizar estimativos, el estudiante va emitiendo sus propios juicios respecto a los resultados que va obteniendo, según vaya armando las figuras cónicas en plastilina). Con las respuestas numéricas a esta evaluación solamente se pretende que el estudiante concluya las potencias a las que está elevado el factor de escala, al relacionar entre sí; longitudes, áreas, y volúmenes. La pregunta uno, tiene varias preguntas que llevan al estudiante a deducir el factor de escala que relaciona las áreas laterales de dos conos de diferente tamaño y sus respectivos volúmenes. Dado que este proceso ya se había realizado para otras figuras geométricas similares, los estudiantes no tuvieron problema en su solución, por esto el total de los estudiantes respondió correctamente a todas y cada una de las preguntas de este apartado. La pregunta 2 repite el proceso anterior con un cono de distinto tamaño, pero en menos pasos. En este ítem, algunos estudiantes se equivocaron en algunas de las respuestas parciales, por eso no se les asignó respuesta correcta; sin embargo, en la siguiente pregunta al variar nuevamente las dimensiones del cono, haciendo menos preguntas y llegando de forma directa a la relación entre volúmenes y entre áreas, el porcentaje de respuestas correctas fue mayor. Este 
avance implica que salvo algunos errores aritméticos que cometen, el proceso se asume como asimilado por los estudiantes. Los resultados obtenidos en esta evaluación se muestran en la gráfica de la figura 10.

Figura 10

Resultados evaluación guía 3

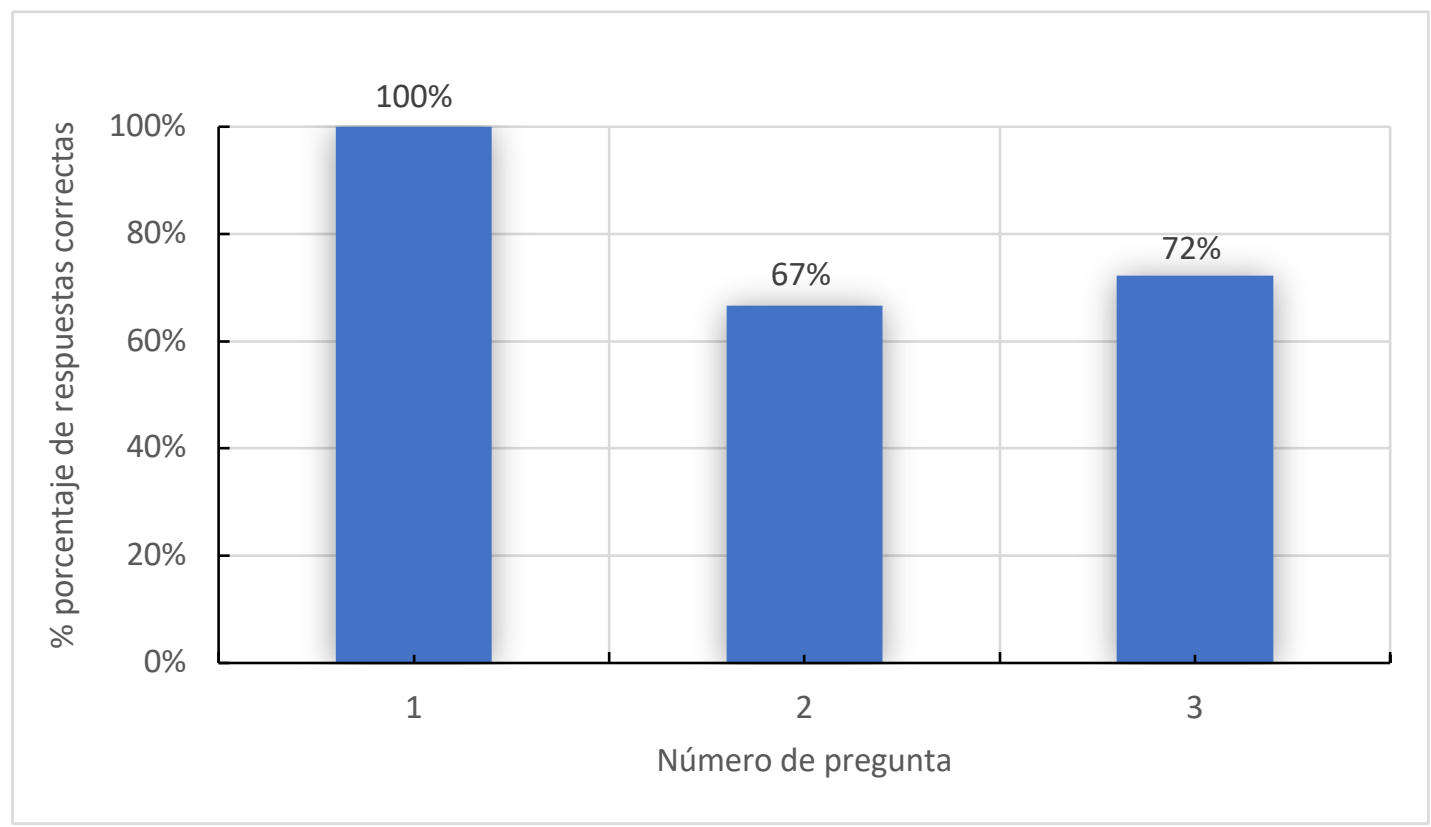

Fuente: (Villa Celedon, 2020)

Con base en lo anterior podemos deducir porque el promedio del curso fue de 2.4/3.0, lo cual equivale a 8.0/10.0, que es un buen promedio, porque tiene en cuenta todas las respuestas dadas por los estudiantes en los tres numerales en que se resumió esta evaluación (Villa Celedon, 2020).

En la evaluación de la guía 4, sobre leyes isométricas y aplicaciones, se retomó el ejemplo de la división celular, asignándole un valor numérico al tamaño de las células con la finalidad de indagar si los estudiantes podían llegar a conclusiones semejantes. Con respecto a la relación entre las superficies de las células y entre sus volúmenes (esferas), pero procurando que llegarán a la conclusión esperada: la relación entre las necesidades máximas de oxígeno que pueden adquirir las células madre e hija respectivamente y finalmente, a la relación entre los factores de viabilidad de las células madre e hija también.

De los resultados mostrados en la figura 11, es evidente que, con respecto a la guía anterior, el proceso se asimiló de forma más significativa, ya que, en las primeras tres partes que tienen que ver con el factor de escala entre radios, área y volúmenes, el $100 \%$ respondieron correctamente. En lo que tiene que ver con los factores de viabilidad, lo respondieron correctamente en un $61 \%$, es decir 7 estudiantes tuvieron dificultades, pese a esto, los demás estudiantes respondieron en forma correcta; al parecer, la duda mayor radicó en si el factor de viabilidad aumentaba o disminuía en las células más viejas, ya que esto no pareció muy evidente para ellos. 
Figura 11

Resultados evaluación guía 4.

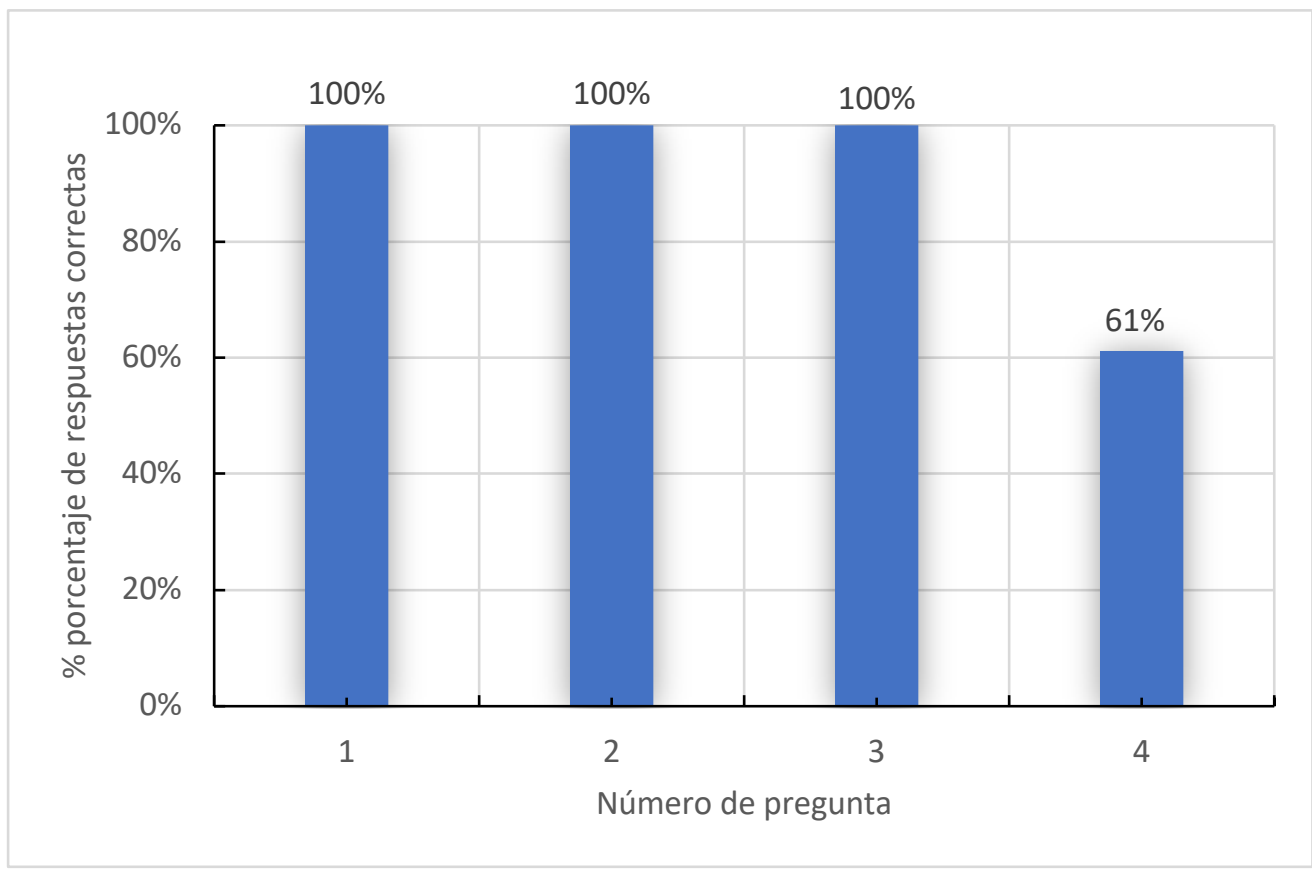

Fuente: (Villa Celedon, 2020)

Si se tiene en cuenta que el promedio general del grupo en toda la evaluación correspondió a 9.0/10.0, es un resultado bastante bueno, esto evidencia que será necesario seguir insistiendo en lo que tiene que ver con las aplicaciones concretas (Villa Celedon, 2020).

En lo que concierne a la evaluación de la quinta guía, sobre el Modelo de Semejanza Elástica, las Leyes Alométricas y la Ley de Kleiber, se tomó el ejemplo de la comparación entre las magnitudes (tamaños y masas) de una persona adulta y un niño, asignándole valores numéricos a las variables (aquí no hay que olvidar que los estudiantes pueden equivocarse al realizar los cálculos numéricos, lo cual no necesariamente significa que no hayan entendido los conceptos, pero sí es un punto en el cual hay que prestar atención). Esta evaluación tuvo cinco partes: en la primera se exploró sobre la aplicación del Modelo de Semejanza Elástica para encontrar la altura del adulto, conociendo la altura del niño, asumiendo que sus cuerpos cuentan con forma cilíndrica. En la segunda, utilizando la Ley Alométrica que relaciona la masa con el diámetro de los cilindros, se pregunta la masa del adulto conociendo la masa del niño; en la tercera, utilizando la Ley Alométrica que relaciona la superficie corporal y la masa, se pide realizar los cálculos de las superficies de los cuerpos del niño y del adulto, suponiéndolos con forma cilíndrica y conociendo sus masas. La cuarta, de forma intencional se sale un poco del esquema de las anteriores, ya que, se cuestiona al estudiante sobre aproximaciones se han realizado al aplicar las relaciones alométricas que se utilizaron en cada caso. Esta pregunta de tipo conceptual tiene la finalidad de explorar si el estudiante es consciente de que estos cálculos no son del todo exactos, sino que hay algunas aproximaciones implícitas en el uso de las relaciones alométricas, comenzando simplemente por la suposición de cuerpos humanos con forma de cilindro. La quinta pregunta, enfocada hacia la aplicación de la Ley de Kleiber, pide realizar los cálculos pertinentes para hallar la $T M B$ del adulto. Más allá del cálculo solicitado, en el proceso se está recordando en cada momento el significado biológico y físico de cada uno de los términos. Este es un refuerzo conceptual que más que evaluar, ayuda a interiorizar las relaciones biológicas y físicas utilizadas. Las conclusiones esperadas de los estudiantes en esta guía están relacionadas con el manejo del Modelo de Semejanza Elástica, las Relaciones Alométricas y la Ley de Kleiber. 
Figura 12

Resultados evaluación guía 5

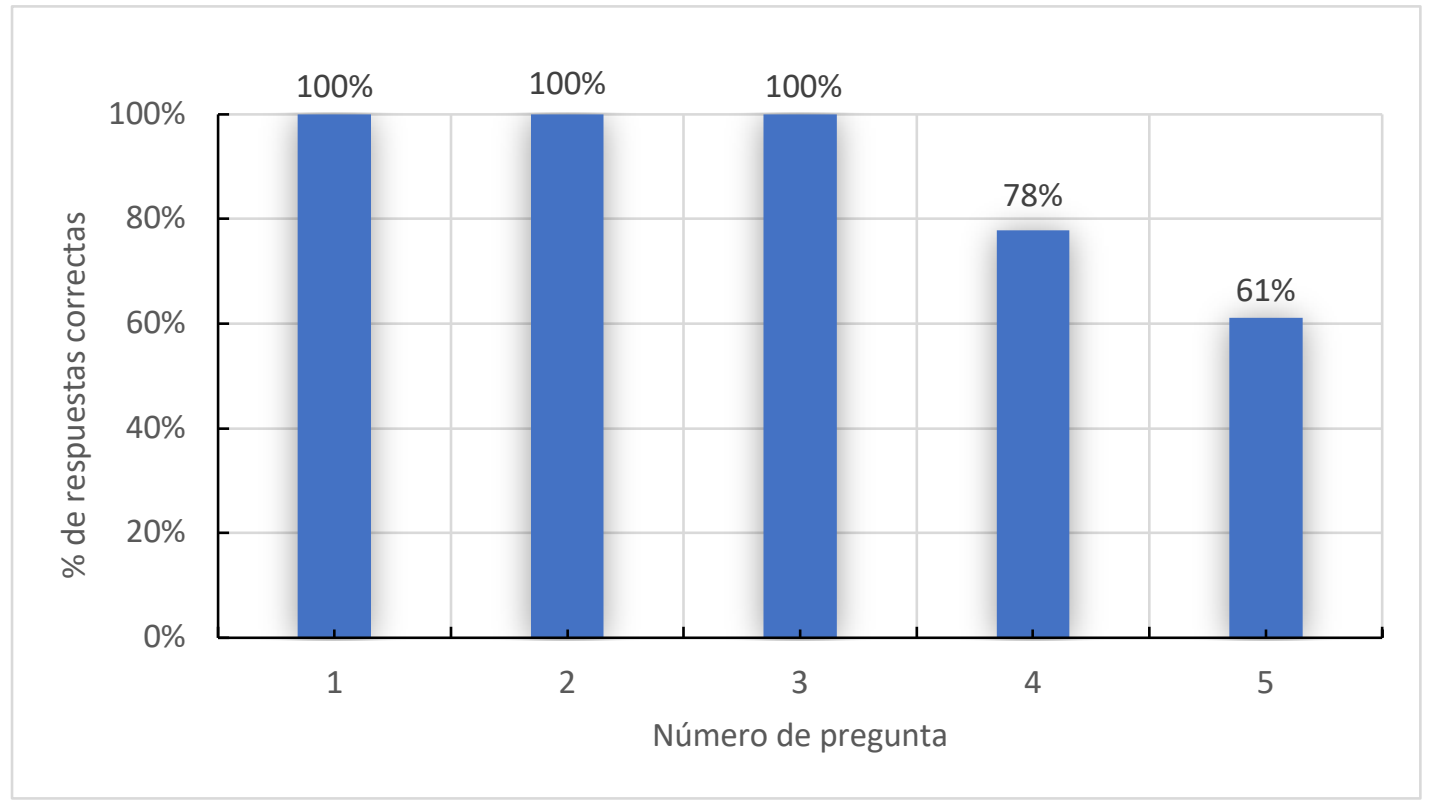

Fuente: (Villa Celedon, 2020)

En las primeras tres preguntas los estudiantes no tuvieron ningún problema en resolverlas correctamente, ya que a medida que se les solicitaba la realización de cálculos aritméticos, se les iba guiando en el manejo de la calculadora, ya que para ellos no es inmediato resolver potencias fraccionarias como por ejemplo $2,965^{\frac{5}{8}} \mathrm{y}$ realizar operaciones con ellas. Esto se evidencia porque el $100 \%$ de los estudiantes respondieron bien a las tres primeras preguntas.

La cuarta pregunta que es totalmente teórica y se podría afirmar que en general no obtuvo una respuesta muy acertada (78\%), probablemente porque la mayoría de los estudiantes contestaron que "...se pueden relacionar los cuerpos geométricos a la mayoría de los animales o personas tales como la esfera o cilindros, pero no sabíamos que al cambiar una medida también se alteraban las otras para conservar su igualdad..."; sin embargo, ese tipo de afirmaciones deja ver que los estudiantes ya están entendiendo que pueden establecer relaciones entre figuras geométricas simples y la morfología de algunos seres vivos.

La quinta pregunta (que presenta cálculos numéricos) fue la que menos respuestas correctas tuvo (fallaron en alguna parte de la respuesta 7 estudiantes de 18, es decir el 61\%), posiblemente debido, a que algunos de ellos aún no relacionan muy bien la aritmética con algunas funciones o conceptos de la biología.

El promedio aritmético general de todo el curso para esta prueba fue de 8.4/10.0, lo cual deja entrever el avance de los estudiantes, ya que esta última guía conjuga el tratamiento aritmético y algunos conceptos elementales de Física y de Biología, interdisciplinariedad a la cual no están muy acostumbrados la mayoría de los estudiantes (Villa Celedon, 2020).

Como se mencionó en el apartado anterior, al terminar de desarrollar todas las guías con su respectiva evaluación y retroalimentación de manera virtual, se procedió a la aplicación de la evaluación final.

De los resultados obtenidos en esta evaluación, es claro que los estudiantes ya están, no solamente familiarizados con el tema de Factores de Escala, Leyes Isométricas, Leyes Alométricas (y algunas aplicaciones), sino también con los conceptos involucrados en la Ley de Kleiber. Este avance se puede deducir del $100 \%$ de 
respuestas correctas a las primeras 7 preguntas, ya que estas evaluaron los temas de las primeras cuatro guías. La pregunta 8 evaluó factores de escala en cilindros, mientras que la pregunta 9 evaluó fuerza relativa y la pregunta 10 evaluó una aplicación de la TMB. La mayoría de los errores que causaron falla en la respuesta a las últimas preguntas se atribuyeron a errores de cálculo numérico, por ejemplo, en la pregunta 10, la mitad de los estudiantes no calculó bien el valor de $8^{\frac{4}{3}}=16$.

La figura 13 muestra los resultados de la evaluación final.

Figura 13

Resultados evaluación final

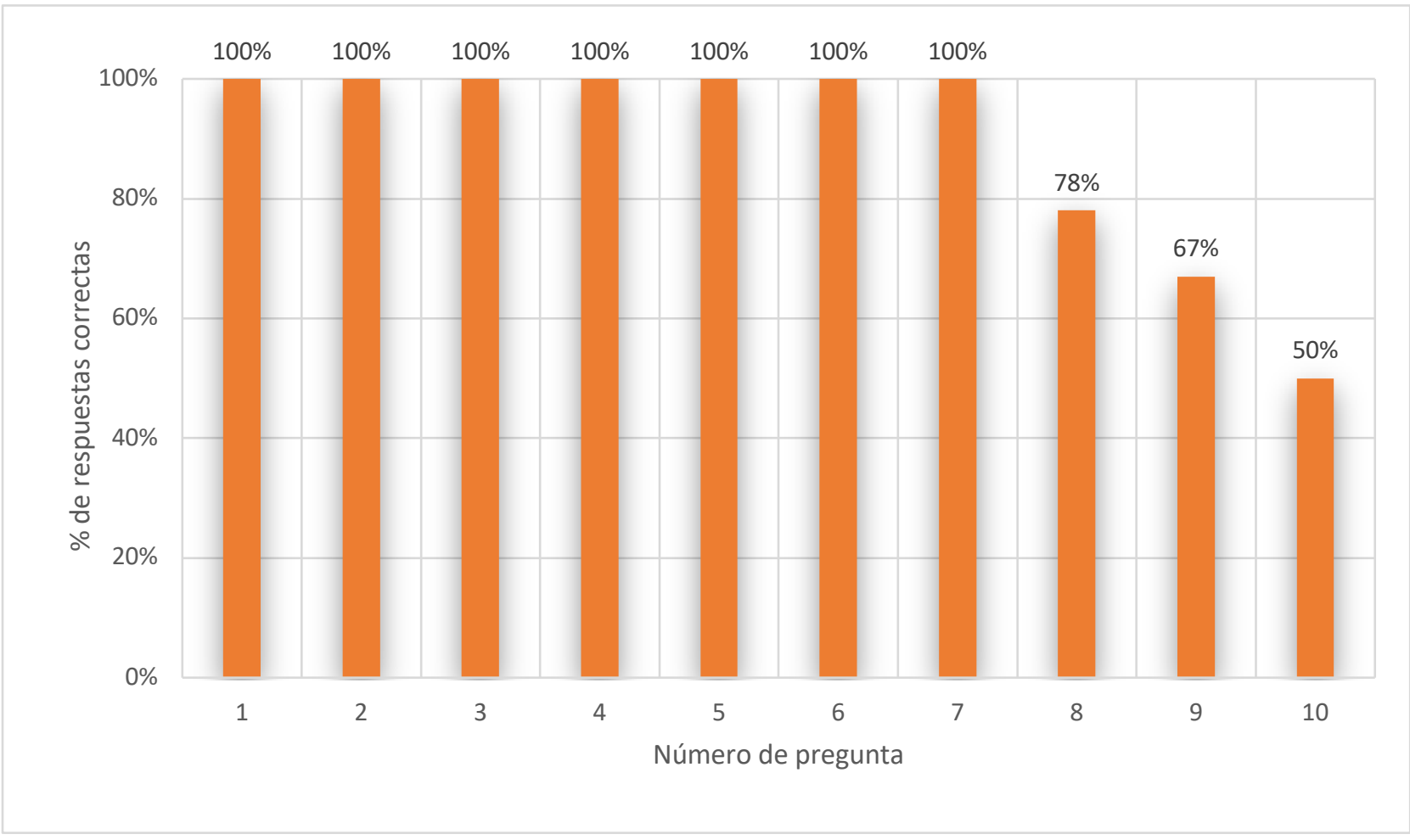

Fuente: (Villa Celedon, 2020)

El promedio aritmético general del grupo en la evaluación final fue de 9.0/10.0, es decir que en promedio, se presentó un $90 \%$ de aciertos correctos (Villa Celedon, 2020).

Para comparar los resultados de las evaluaciones inicial y final, se tomaron aquellas preguntas en las que se indagaron los mismos temas: manejo de variables, cálculo de volúmenes, regla de tres en fuerza relativa, distribución y cálculo de volúmenes y regla de tres entre masa y volumen.

En la figura 14 se muestra el gráfico que permite comparar los resultados obtenidos en la evaluación inicial y final. 
Figura 14

Gráfico comparativo entre los resultados de las evaluaciones inicial y final en las preguntas que exploraron los mismos temas, con preguntas similares pero no idénticas.

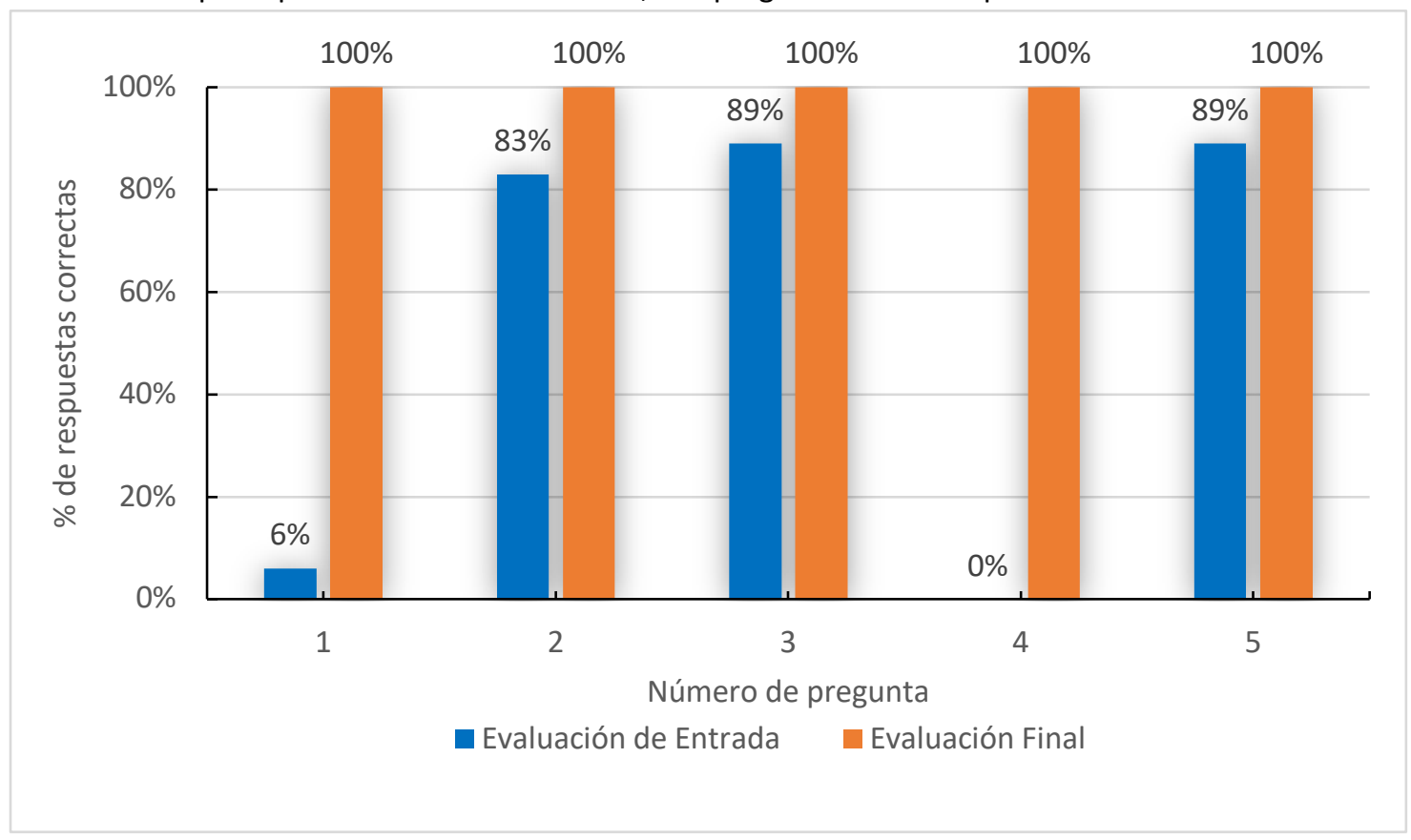

Fuente: (Villa Celedon, 2020).

Una observación superficial de la gráfica anterior, da la idea del gran avance en los resultados obtenidos en estas preguntas entre la evaluación inicial y la evaluación final.

Es de resaltar el notable avance en las respuestas dadas a las preguntas 1 y 4 , ya que eran muy pocos los estudiantes que las habían respondido en la evaluación inicial, pero tranquiliza que no pertenecían a los temas centrales o no entendidos, antes bien, corresponden a solución de reglas de tres simple directa, de donde se infiere que nuevamente las fallas pueden corresponder a dudas en el manejo de la calculadora (Villa Celedon, 2020).

Para cuantificar el avance entre estas dos evaluaciones, acudimos al Factor de Ganancia Normalizada de Hake $(G N H)$, el cual es un factor que sirve para comparar resultados entre pruebas análogas (para el caso, solo entre las cinco preguntas que indagaban sobre los mismos temas en ambas evaluaciones). El GNH se define como el cociente entre la diferencia porcentual de respuestas correctas entre las pruebas de entrada y salida y el máximo valor posible obtenible por el estudiante (Hake, 1998):

$$
\langle g\rangle=\frac{\%\left\langle s_{f}\right\rangle-\%\left\langle s_{i}\right\rangle}{100-\%\left\langle s_{i}\right\rangle}
$$

Donde $\langle g\rangle$ es el $G N H, \%\left\langle s_{i}\right\rangle$ es el promedio porcentual de respuestas correctas de la prueba inicial y $\%\left\langle s_{f}\right\rangle$ es el promedio porcentual obtenido en la prueba final. El GNH calculado mediante la ecuación (5), permite categorizar los resultados obtenidos por los estudiantes en tres zonas, así:

$$
\begin{array}{lll}
\text { Si } & \mathbf{0}<\langle\boldsymbol{g}\rangle \leq \mathbf{0 , 3} & \text { Ganancia baja } \\
\text { Si } & \mathbf{0 , 3}<\langle\boldsymbol{g}\rangle \leq \mathbf{0 , 7} & \text { Ganancia media }
\end{array}
$$




\section{Si $\quad \mathbf{0 , 7}<\langle\boldsymbol{g}\rangle \leq \mathbf{1 , 0} \quad$ Ganancia alta}

Al realizar los respectivos cálculos para todos los estudiantes en ambas evaluaciones, se obtuvo $\%\left\langle s_{i}\right\rangle=52 \%$ y $\%\left\langle s_{f}\right\rangle=90 \%$ y utilizando la ecuación (5), se obtuvo $\langle g\rangle=0,79$, que ubica los resultados de la comparación entre las dos evaluaciones en el rango de ganancia alta, lo cual se podía prever, según se analizó en los resultados de la evaluación final.

\section{Conclusiones}

\section{Respecto al tema enseñado}

Aunque el tema seleccionado para enseñar (Factores de Escala) está contemplado en los Estándares Básicos de Competencias en Ciencias y Matemáticas del Ministerio de Educación Nacional de Colombia (MEN), en el documento se presenta de forma aislada; sin que entre los conceptos de Aritmética y Geometría se establezca relación con los de Física y Biología. Es claro que, aunque, inicialmente el tema era desconocido para los estudiantes, al final lograron asimilarlo en una gran medida. Por lo tanto se puede inferir, que la pertinencia de su estudio fue, es y será bastante acertada con los estudiantes de secundaria.

\section{Respecto a la interdisciplinariedad}

Como lo manifestaron algunos estudiantes en las conclusiones de varias de las prácticas desarrolladas, ellos no se imaginaban que para estudiar algunos temas de Biología, por ejemplo, tuviesen que utilizar la Aritmética y la Geometría, afirmaron que: "...no se imaginaban que hubiese ese grado de interdisciplinariedad entre estas áreas..." Con ninguno de sus profesores había trabajado de esta forma: "...al parecer las matemáticas tienen muchas más aplicaciones de las que nos imaginábamos...". Estas reflexiones son muy dicientes en estudiantes de este nivel, ya que, si pudiesen seguir desarrollando muchos temas interdisciplinariamente encontrarían que las Ciencias Naturales y las Ciencias Exactas son complementarias en más fenómenos y descripciones de la naturaleza de los que ellos se imaginan, de tal forma que cuando lleguen a los grados superiores y vean los cursos de Física y Química, encontrarán mucha relación con los contenidos que se les orienten en Geometría elemental, Geometría analítica, Trigonometría y Cálculo y podrían identificar a las ciencias exactas, no solamente como una forma cuantitativa de describir algunos fenómenos de la Física o la Biología, sino también como un lenguaje más preciso de comunicación entre ellas.

\section{Respecto a la metodología empleada (MAA-MV)}

Al implementar en la MAA las modificaciones aquí descritas para mantener el aprendizaje activo a pesar del cambio de la presencialidad a la virtualidad, fue necesario tener "contacto virtual" con los estudiantes, de una forma más contínua y permanente. Asimismo fue importante tener un control más cercano realizando retroalimentaciones precisas, debido a que a pesar de que en sus hogares podían tener ayuda, no siempre contaban con ella o en algunas ocasiones no era la orientación más apropiada (los padres de estos estudiantes no todos tienen alta formación académica). Por otra parte, evidenció que la temática y la misma metodología los motivó al trabajo en casa, pero solo después de comenzar a realizar experimentos con papel y plastilina, ya que el solo cálculo aritmético de áreas y volúmenes inicial no los motivó. Los mismos estudiantes al final solicitaban que en las otras materias se siguieran metodologías similares que los motivaran y les permitieran un trabajo más experimental e interdisciplinar, para encontrarle mayor gusto a los temas a pesar de la virtualidad. 


\section{Respecto a la forma de evaluar}

El hecho de haber realizado una evaluación permanente (evaluación al final de cada guía y por medio de la proposición de situaciones similares a las estudiadas en la guía), además de la evaluación sumativa, arrojó buenos elementos para la asimilación de los temas y una visión más amplia del avance paulatino de los estudiantes. Es correcto pensar que este tipo de acciones proporciona elementos de juicio para evaluar el avance de los estudiantes en comparación con la evaluación sumativa (esta última realizada al final por medio de una evaluación en test con pregunta de selección múltiple con única respuesta, en donde no se sabe si los errores son debidos a cálculos aritméticos o conceptuales); sin embargo, es de anotar que ambos tipos de evaluación (formativa y sumativa) se complementaron muy bien, dando luces bastante concretas de los avances y resultado final obtenido por los estudiantes.

\section{Respecto a los resultados}

Como se pudo observar en el apartado de resultados, en las primeras evaluaciones, incluyendo la evaluación inicial o diagnóstica, y al finalizar las primeras guías, los resultados no fueron los mejores, comenzaron con un promedio de 5.2/10.0 y finalizaron con un promedio de 8.4/10.0 y 9.0/10.0 en la última guía y en la evaluación final respectivamente. Esto muestra claramente el avance de los estudiantes en la obtención de buenos resultados. Más allá del mejoramiento numérico de los resultados, el mayor logro se evidenció cuando en las sesiones de retroalimentación de las evaluaciones de las guías y de la evaluación final, los estudiantes manifestaban y mostraban una actitud mucho más positiva y propositiva con respecto a la temática estudiada que al principio; así como una transformación en su discurso, pues hicieron uso de algunos conceptos, hablaron apropiadamente de las Leyes Isométricas, de las Leyes Alométricas y de la Ley de Kleiber. En resumen, aunque los resultados numéricos hubiesen podido ser mejores, se considera que bajo las circunstancias en las que se desarrolló el trabajo, en el cual se hizo una planeación de trabajo experimental totalmente presencial y luego, sobre la marcha fue necesario cambiar algunos pasos de la $M A A$, por una $M A A-M V$. Estos ajustes no generaron inconvenientes mayores, antes bien, se convirtieron en un factor motivante, ya que aunque habían tiempos de entregas de evaluaciones y asesorías, los estudiantes se sintieron motivados al ver que cosas tan importantes como la cantidad de energía necesaria para la sobrevivencia de un ser vivo, pudiese ser cuantificada y también ver cómo las Leyes Alométricas podrían de alguna forma describir algunos cambios en la anatomía de los seres a medida que van creciendo y que podían aproximar el fenómeno con figuras geométricas sencillas; es decir, que para algunos, de alguna manera "...la geometría cobra vida...", tal y como lo afirmó uno de los estudiantes en una de las tutorías. Por otra parte, los estudiantes pudieron manejar sus propios tiempos de trabajo y en algunos casos, por su misma iniciativa, buscar lecturas adicionales de complemento.

Al finalizar el desarrollo del presente trabajo se pudieron evidenciar varias fortalezas, entre ellas:

Se logró una interconexión importante entre temáticas de diferentes áreas (la parte operativa de algunos conceptos de matemáticas y de geometría con la aplicación de algunos conceptos de Física y Biología elemental), que no está contemplada en los lineamientos del MEN.

La metodología experimental utilizada (a pesar de la obligada virtualidad), en definitiva, motivó a los estudiantes orientados, al estudio de temas que anteriormente para ellos eran completamente indiferentes.

La pertinente combinación de la evaluación permanente y sumativa al inicio, a lo largo y al final del proceso, permitió monitorear los avances, realizar a tiempo los refuerzos pertinentes y adquirir una visión más ajustada de la adquisición de los conocimientos y destrezas de los estudiantes. 


\section{Referencias}

Gould, S. J. (1966). Allometry and Size in Ontogeny and Phylogeny. Biological Reviews, 41: 587 - 640.

Gutierrez Sanchez, J. L., \& Sanchez Garduño, F. (Octubre-Diciembre de 2017). Matemática del crecimiento orgánico. Revista de Cultura Científica (126): 76-78.

Guzman Peña, A. (2019). Leyes de Escala: tamaño, forma y vida. Valladolid: Trabajo fin de grado (Grado en Física).

Hake, R. R. (1998). Interactive-engagement versus traditional methods: A six-thousand-student survey of mechanics test data for introductory physics courses. American Journal of Physics, Volume 66, Issue 1: 64 74.

Hudson, L. N., Isaac, N. J., \& Reuman, D. C. (2013). The Relationship Between Body Mass and Field Metabolic Rate Among Individual Birds and Mammals. Journal of Animal Ecology, 82, 1009 - 1020.

Hulbert, A. J., \& Else, P. (2004). Basal Metabolic Rate: History, composition, regulation and usefulness. Physiological and Biochemical Zoology, 77(6): 869 - 876.

Lakhdar, Z. B., Culaba, I. B., Lakshminarayanan, V., \& Maquiling, J. T. (2006). Active Learning in Optics and Photonics: ALOP: manual de entrenamiento (págs. 10-20). Editor: David Sokoloff, University of Oregon. UNESCO.

Mirth, C. K., Frankino, W. A., \& Shingleton, A. W. (2016). Allometry and size control: what can studies of body size regulation teach us about the evolution of morphological scaling relationships? Current Opinion in Insect Science(13), 93-98.

Nuñez, P., Calderon, S. E., \& Gil, S. (2010). Búsqueda de orden y armonía en la naturaleza, descubriendo leyes de escala en el aula. Latin-American Journal of Physics Education, Vol. 4, No. 1, 118 - 126.

Perez, O., \& Fernandez, P. (2015). Leyes de Escala Aplicadas a las Ciencias Biológicas. Universidad de Panamá. Ciudad de Panamá: Facultad de Ciencias Naturales, exactas y tecnología.

Pogorelov, A. V. (1974). Geometría Elemental. Moscú: MIR.

Sanchez Garduño, F., \& Gutierrez Sanchez, J. L. (2020). La alometría, una ley de potencias ubicua en la estructura de los seres vivos. INTERdisciplina 8, No. 20, 11 - 22.

Sokoloff, D., \& Thornton, R. (1997). Using Interactive Lecture Demonstrations To Create An Active Learning Environment. The Physics Teacher, Vol. 35; 340 - 345.

Villa Celedon, O. (2020). Unidad didáctica para la enseñanza-aprendizaje de las leyes de escala, como estrategia interdisciplinar en octavo grado, utilizando la Metodología de Aprendizaje Activo. Universidad Nacional de Colombia. Bogotá: Facultad de Ciencias.

Zaballos, J. P., Diaz, J. A., \& Garcia Moreno, A. (2009). Modelos Adaptativos en Zoología (Manual de prácticas), 2. Tamaño, Forma y Alometría. Reduca (Biología). Serie Zoología, 2(2): 20 - 30.

Esta obra está bajo una Licencia Creative Commons Attribución-NoCommercial 4.0 International

$(\mathrm{Cc})$ BY-NO 